(C) 2018 IEEE. Personal use of this material is permitted. Permission from IEEE must be obtained for all other uses, in any current or future media, including reprinting/republishing this material for advertising or promotional purposes, creating new collective works, for resale or redistribution to servers or lists, or reuse of any copyrighted component of this work in other works. doi: 10.1109/PESGM.2018.8586406

Publisher version: https://ieeexplore.ieee.org/document/8586406

\title{
Linear and Uniform System Dynamics of Future Converter-Based Power Systems
}

\author{
David Raisz, Aysar Musa, Ferdinanda Ponci, Antonello Monti \\ Institute for Automation of Complex Power Systems, E.ON Energy Research Center \\ RWTH Aachen University, Mathieustraße 10, 52074 Aachen, Germany \\ \{draisz, amusa, fponci, amonti\}@eonerc.rwth-aachen.de
}

\begin{abstract}
The share of power converter based renewable generation is steadily increasing at the expense of rotating inertia. Virtual Synchronous Machines are deemed to ensure system stability, however, there are no widely accepted criteria for the design of their controllers. In converter-dominated power systems one of the main challenges will be to analyze large-signal stability, because of the large number (and possibly divergent design) of converters. Converters, however, also offer the possibility to shape system dynamics in a way that was impossible with synchronous machines. In this paper we introduce the new concept of Linearized and Uniform Swing Dynamics. This allows to linearize the nonlinear swing equation over almost the entire state space, thereby enabling to use the small-signal stability analysis technique for large signal stability investigations. By properly choosing the controller parameters, the dynamics of the large number of converters expected in the system can be made unified and predictable.
\end{abstract}

Index Terms-- Converters, Power system control, Power system dynamics, Power system stability, Renewable energy sources

\section{INTRODUCTION}

The increasing share of electricity from renewables (mainly wind and solar) leads to a growing number of converters connected to the power system. Scenarios exist for $100 \%$ renewable-based energy supply in Germany by 2050 [1].

Several research projects (e.g. [2], [3]) therefore aim at the proper establishment of requirements for the design and control of these converters.

Inertia-less system operation is one of the critical challenges that future power systems face. This is due to the significant replacement of synchronous generation with power electronicbased Renewable Energy Systems (RES). In such systems, disturbances will induce new types of dynamic behaviour, which are faster than conventional power system dynamics. These might lead to instability problems and have to be considered in system operation, stability analysis, and control [4], [5].

Moreover, the number of generating units is increasing by several orders of magnitude, as compared to the "conventional" power systems, for which stability concepts are well established. Without a coherent design of inverters and their controllers, power system dynamics will be very hard to

This work has been funded by the European Commission through the Horizon 2020 research and innovation programme, under the RESERVE project grant agreement No. 727481.

The authors gratefully acknowledge funding by the German Federal Ministry of Education and Research (BMBF) within the Kopernikus Project ENSURE

'New ENergy grid StructURes for the German Energiewende'. predict and analyze.

The European RESERVE project investigates the above topics, under the task of frequency stability and control of power systems with up to $100 \%$ RES [6]. One of the main factors is system swing dynamics, which are driven and influenced by the behaviour of existing Synchronous Generators (SG), and are represented by the so called swing equation.

The Swing Equation (SE) is a mathematical form that represents the mechanical dynamics of a SG and is interpreted by the power-angle characteristics. The SE is used for stability analysis, frequency control and regulation. The original nonlinear form of SE interprets the nonlinearity caused by the electromechanical oscillations in SG, and causes most of the complexity in stability analysis and control design [4], [7]. Therefore the SE is usually linearized with specific assumptions around an operating point to simplify the above processes, but then it is applicable only for small disturbances, in which small signal stability is performed to capture system stability information for limited operation conditions. It is worth mentioning that in a future system with $100 \%$ nonsynchronous generation, i.e. power electronic-based RES, the concept of swing equation will be changed, as there are no electromechanical oscillations without SG.

The up-to-date solutions for the integration of power electronic-based RES is the emulation of SG as a Virtual Synchronous Generator (VSG), also referred to as Virtual Synchronous Machine (VSM), see [4], [8], [9], [10].

However, these VSMs are based on the classical representation of SE, which does not make sense in future systems where all SGs are replaced with power electronic-based RES. Hence, an efficient form of SE should be represented and considered in the control design of VSMs.

With this paper we aim to contribute to the establishment of the requirements for the future, power converter-based power systems. We are doing so by proposing the concept of Linear and uniform Swing Dynamics (LSD) which aims to tackle the nonlinearity of SE and provides: linear dynamical system, enhanced system stability and uniform and predictable dynamic performance. This concept enables to perform system stability analysis and capture information for the whole state space, i.e. small and large disturbances, as well. Based on this concept, this work proposes an Improved VSM, which achieves linear 
power-angle characteristics by introducing specific modification in the control design. Simulation results are presented to demonstrate LSD controller operation.

\section{POWER SYSTEM SWING DYNAMICS: TRENDS AND SOLUTIONS}

\section{A. Power system swing equation}

The swing equation expresses the mechanical dynamics of a synchronous machine, due to any imbalance between the electromagnetic and mechanical torque. It is represented by:

$$
J \frac{d \omega_{m}}{d t}=J \frac{d^{2} \theta_{m}}{d t^{2}}=T_{a}=T_{m}-T_{e}-T_{d}
$$

where $J$ : combined moment of inertia (prime mover and generator), $\omega_{m}$ : angular velocity, $\theta_{m}$ : angular displacement of rotor, $T_{a}$ : accelerating torque, $T_{m}$ : prime mover torque, $T_{e}$ : electrical counter torque, $T_{d}$ : damping torque.

Note that the relation between electrical and mechanical quantities depends on the number of poles $(p)$, as given by $\delta=$ $\frac{p}{2} \delta_{m}$ and $\omega=\frac{p}{2} \omega_{m}$, where $\theta_{m}=\omega_{m}+\delta_{m}, \theta=\omega+\delta$

Assuming $p=2$, the resulting swing equation in terms of electrical power angle $\delta$ will be

$$
\begin{gathered}
M \frac{d^{2} \delta}{d t^{2}}=P_{m}-P_{e}-P_{d} \\
M=\frac{2 H}{\omega}, \omega=2 \pi f
\end{gathered}
$$

$M$ :moment of inertia, $\mathrm{H}$ : inertia constant, and $f$ : system frequency

The swing equation is used to analyse system transient stability, using the equal area criterion [6],[7]. Also, it is used in frequency control design and regulation.

The well-known power angle characteristic is used in the transient stability analysis, and is expressed as follows:

$$
P_{e}=\frac{E * V}{X} \sin \delta
$$

where $E$ is the connection bus terminal voltage, $V$ is the generator terminal voltage, and $X$ is the grid impedance, assuming $\mathrm{X} \gg R$.

From (3), a nonlinear relationship is observed between machine's active power $\left(P_{e}\right)$ and rotor angle $(\delta)$. This nonlinearity interprets the nonlinear swing dynamics in power systems which is influenced by the behavior of connected SM within power system. The nonlinear swing dynamics pose critical constraints and limitations to system stability analysis and control design. However, in future power systems with very high share of RES and less SMs, new concepts can be introduced to the above-explained nonlinear relationship. In this regard, the Authors propose the concept of Linear Swing Dynamics (LSD) that can be embedded in the control of REStied inverters of future power systems, as discussed in Section III.

\section{B. Trends and Solutions}

To adapt securely the integration of RES with maintaining similar characteristics of conventional SM, the swing equation has been embedded in the inverter control of RES as in [4] or
[10]. The swing equation is realized as the heart of so called Virtual Synchronous Machine (VSM), which aims to emulate inertia provision. A comprehensive overview on VSM concepts can be found in [11] and [12].

\section{Virtual Synchronous Machine (VSM)}

For the purpose of our simulation studies, we chose a VSM concept that has a cascaded control structure for the following reasons:

- $\quad$ to demonstrate the concept, we need a simple design structure

- we will find it convenient to have easy access to the voltage reference signal.

Therefore we are using the VSM concept presented in [13] and [14]. The configuration is shown in Fig. 8. The inverter is connected to the grid via an LC filter. It is assumed that an energy source or storage with sufficient capacity is connected to the DC link, which is thus represented by a voltage source. A nested control loop structure is adopted where current and voltage can be controlled separately. The control strategy is implemented in the synchronously rotating reference frame (SRF), based on well-known PI controllers with feed-forward cross-coupling terms applied to the dq-coordinates in the current-, voltage- and virtual impedance (VI) loops. The swing equation (and not the PLL) provides the frequency and phase angle references to the control system, thereby implementing the virtual inertia behavior. The active power control is provided by an outer steady-state frequency droop controller (see $k \omega$ in Fig. 1.):

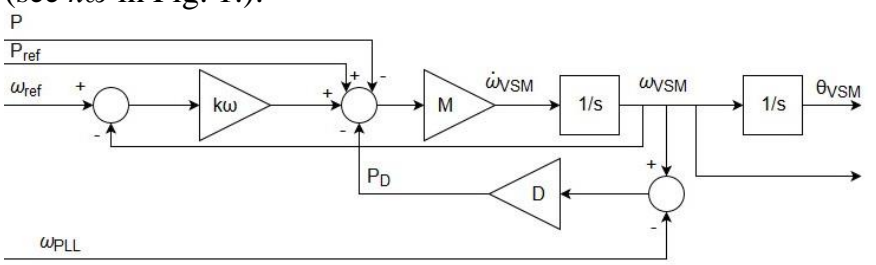

Fig. 1. VSM control block diagram

A reactive power droop contributes to the voltage magnitude reference.

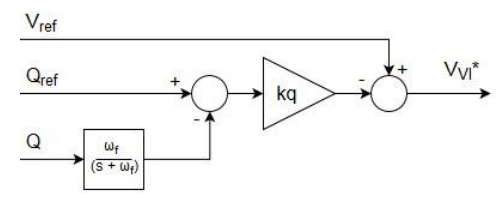

Fig. 2. Reactive power control block diagram

For the sake of simple analysis throughout this paper, we set $k q=0$.

The equations, the control schemes of the current-, voltage and impedance loops, as well as a detailed explanation of the PLL and additional active damping terms are explained in detail in [14].

\section{IMPROVED VIRTUAL SYNCHRONOUS GENERATOR FOR FUTURE INERTIA-LESS SYSTEMS}

Our main contribution is the LSD concept, which can be implemented as 
- $\quad$ either an additional block in the above structure (see dashed lines in Fig. 8)

- $\quad$ or as a special design of the VI.

In the first case, the LSD block provides the voltage reference, based on the active power reference, as described in Chapter III.B.1). In the second case, the LSD block is omitted, and the VI values have to be calculated as shown in Chapter III.B.2).

\section{A. Linear Swing Dynamics (LSD)}

In order to derive the LSD concept, first we analyze the simplified system shown in Fig. 3
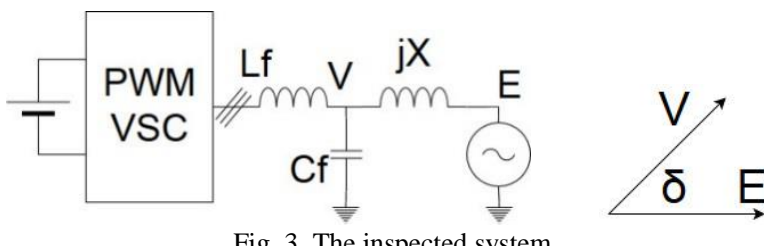

Fig. 3. The inspected system

It consists of a three-phase PWM controlled VSC, interfaced to a Thévenin-equivalent system by an LC-filter.

The following swing equations (recall (1) - (3)) govern VSM dynamics:

$$
\begin{gathered}
M \dot{\omega}=P-P_{e}-D \dot{\delta} \\
P_{e}=\frac{E V}{X} \sin \delta \\
\dot{\delta}=\omega-\omega_{s}
\end{gathered}
$$

with

$M$ : inertia constant,

$\omega$ : rotational speed,

$\omega_{s}:$ synchronous speed,

$P$ : output power setpoint,

$P_{e}$ : actual inverter output,

$E$ and $X$ : (nominal) voltage and reactance of the equivalent system,

$V$ : inverter output voltage,

$\delta$ : angle between $\mathrm{E}$ and $\mathrm{V}$,

$D$ : damping constant.

Linearizing (4) - (6) around an operating point, we obtain (7) that describes the small-signal behavior of the VSM:

$$
\left[\begin{array}{c}
\Delta \dot{\delta} \\
\Delta \dot{\omega}
\end{array}\right]=\left[\begin{array}{cc}
0 & 1 \\
-\frac{E V}{X M} \cos \delta & -\frac{D}{M}
\end{array}\right]\left[\begin{array}{l}
\Delta \delta \\
\Delta \omega
\end{array}\right]
$$

The dynamic behavior of this system depends on the actual operating point $(\mathrm{Pe})$, and has a nonlinear dependence on $\delta$.

Our first goal is to linearize (5) with respect to $\delta$. Since $E$ and $X$ are constant, this can only be done by an appropriate control of $V$ (considering that $V$ usually has to be within a $5 \%$ or $10 \%$ tolerance band around the nominal voltage).

\section{B. Proposed solutions to achieve LSD}

\section{1) Voltage control-based LSD}

The control law can be derived by solving (8) for $V$ :

$$
P=\frac{E V}{X} \sin \delta=\frac{E}{X}(1-\varepsilon) E \delta
$$

where $\varepsilon$ is the voltage tolerance, usually $0.1 \mathrm{pu}$. for distribution networks and $0.05 \mathrm{pu}$. for transmission systems.
The solution of (8) will yield

and

$$
\delta(P)=\frac{P X}{(1-\varepsilon) E^{2}}
$$

$$
V(\delta)=\frac{(1-\varepsilon) E}{\sin \delta} \delta
$$

It can be shown that for $\varepsilon=0.1$ and by using (9) and (10) the $P(\delta)$ relationship will be linear in the angle range $\left[0^{\circ}, 62^{\circ}\right]$, the corresponding power range $[0,0.97]$ and voltage range $[0.9,1.1]$.

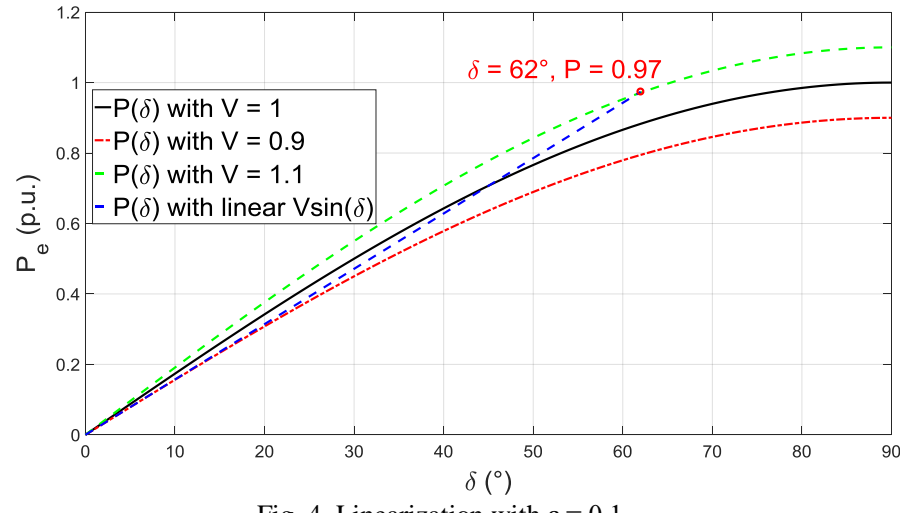

Fig. 4. Linearization with $\varepsilon=0.1$

For $\varepsilon=0.05$ and by using (9) and (10) the $P(\delta)$ relationship will be linear in the angle range $\left[0^{\circ}, 44^{\circ}\right]$, the corresponding power range $[0,0.73]$ and voltage range $[0.95,1.05]$.

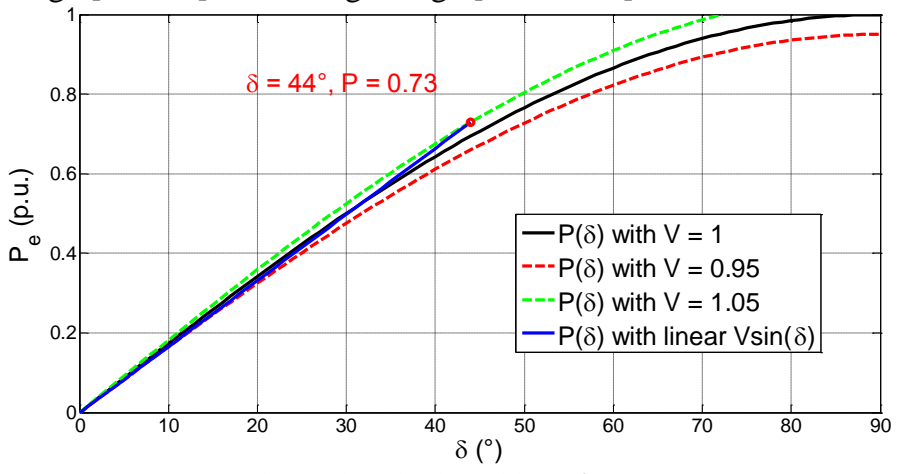

Fig. 5. Linearization with $\varepsilon=0.05$

The control law in (9) - (10) can be achieved by either

- setting $V_{r e f}$ according to (7) and setting the virtual impedance values equal to zero: in this case $V_{\text {ref }}=V_{V I}{ }^{*}=V_{V C}{ }^{*}=V$;

- or keeping $V_{\text {ref }}$ constant, and designing the VI so that the voltage reference input of the voltage control loop $\left(V_{V C}{ }^{*}\right.$ in Fig. 8) be always the same as in (10).

2) Virtual impedance-based LSD

In order to implement the linearization by the conventional virtual impedance technique, let us denote $K=V_{\text {ref }} / E$.

We obtain $\delta$ from (9) and $V$ from (10). Then we calculate the inverter current

$$
I=\frac{V-E e^{-j \delta}}{j X}
$$

and therefore the VI can be calculated as

$$
Z_{v}=\frac{V_{r e f}-V}{I}=\frac{K E-V}{I}
$$


This yields the results (17) and (18) for $R_{v}$ and $X_{v}$ shown in the Appendix.

\section{LSD based Improved VSM}

For the sake of simplicity, we will use the Voltage controlbased LSD method.

By using (9) and (10) the VSM dynamics will be governed by:

$$
\left[\begin{array}{c}
\Delta \dot{\delta} \\
\Delta \dot{\omega}
\end{array}\right]=\left[\begin{array}{cc}
0 & 1 \\
-\frac{(1-\varepsilon) S_{S C}}{M} & -\frac{D}{M}
\end{array}\right]\left[\begin{array}{c}
\Delta \delta \\
\Delta \omega
\end{array}\right]
$$

where $S_{s c}=E^{2} / X$ is the short-circuit power at the connection point.

The eigenvalues are

$$
-\frac{D}{2 M} \pm \sqrt{\left(\frac{D}{2 M}\right)^{2}-\frac{(1-\varepsilon) S_{s c}}{M}}
$$

In order to achieve uniform eigenvalues and oscillation-free dynamics for all inverters, parameters $D$ and $M$ have to follow (15) and (16):

$$
\frac{D}{2 M}=\gamma(\text { same constant for each inverter })
$$

and

$$
\gamma^{2} M \geq(1-\varepsilon) S_{s c}
$$

\section{Simulation AND RESUlts}

In order to demonstrate the LSD concept, we simulated the system shown in Fig. 8 (Single Machine Infinite Bus - SMIB) with the following parameters (for definitions of these parameters, see [14]):

TABLE I. MODEL PARAMETERS FOR SMIB SIMULATION

\begin{tabular}{|l|c|r|c|}
\hline Rated Voltage & $\mathrm{V}_{\mathrm{S}, \mathrm{LL}, \mathrm{RMS}}$ & 400 & $\mathrm{~V}$ \\
\hline Rated Power & $\mathrm{S}_{\mathrm{b}}$ & 500 & $\mathrm{kVA}$ \\
\hline Rated angular freq. & $\omega_{\mathrm{b}}$ & $2 \pi 50$ & \\
\hline VSM Inertia constant & $\mathrm{T}_{\mathrm{a}}=1 / \mathrm{M}$ & 2000 & \\
\hline $\begin{array}{l}\text { VSM Damping } \\
\text { coefficient }\end{array}$ & $\mathrm{D}$ & 40000 & \\
\hline Current controller gain & $\mathrm{k}_{\mathrm{pc}}, \mathrm{k}_{\mathrm{ic}}$ & $2.0,32.6$ & \\
\hline Voltage controler gain & $\mathrm{k}_{\mathrm{pv}}, \mathrm{k}_{\mathrm{iv}}$ & $20.0,10.0$ & \\
\hline Voltage reference & $\mathrm{V}_{\mathrm{ref}}$ & 1 or LSD & $\mathrm{pu}$. \\
\hline Filter inductance & $\mathrm{l}_{\mathrm{f}}$ & 10 & $\mathrm{mH}$ \\
\hline Filter resistance & $\mathrm{r}_{\mathrm{lf}}$ & 0.163 & $\Omega$ \\
\hline Filter capacitance & $\mathrm{c}_{\mathrm{f}}$ & 0 & $\mu \mathrm{F}$ \\
\hline Grid inductance & $1_{\mathrm{g}}$ & 615.4 & $\mu \mathrm{H}$ \\
\hline Grid resistance & $\mathrm{r}_{\mathrm{g}}$ & 0 & $\Omega$ \\
\hline Grid voltage & $\mathrm{v}_{\mathrm{g}}$ & 1 & $\mathrm{pu}$. \\
\hline Droop gains & $\mathrm{k}_{\mathrm{w}}, \mathrm{k}_{\mathrm{q}}$ & $\mathrm{S}_{\mathrm{b}} /(2 \pi), 0$ & \\
\hline Virtual inductance & $\mathrm{l}_{\mathrm{v}}$ & 0 & $\mu \mathrm{H}$ \\
\hline Virtual resistance & $\mathrm{r}_{\mathrm{v}}$ & 0 & $\Omega$ \\
\hline DC side voltage & $\mathrm{V}_{\mathrm{DC}}$ & 5700 & $\mathrm{~V}$ \\
\hline $\begin{array}{l}\text { Active damping cut- } \\
\text { off freq. }\end{array}$ & $\omega_{\mathrm{AD}}$ & 20 & \\
\hline
\end{tabular}

\begin{tabular}{|l|c|r|l|}
\hline Active damping gain & $\mathrm{k}_{\mathrm{AD}}$ & 0 & \\
\hline Current feed-forward & $\mathrm{k}_{\mathrm{ffi}}$ & 0 & \\
\hline Voltage feed-forward & $\mathrm{k}_{\mathrm{ffv}}$ & 1 & \\
\hline Voltage tolerance & $\varepsilon$ & 0.1 & $\mathrm{pu}$. \\
\hline
\end{tabular}

During the simulation, the active power reference was changed from 0.01 to $0.2,0.4,0.6$ and 0.8 pu. At time instants $4,8,12$ and $16 \mathrm{~s}$, respectively. The simulation results are plotted in the Figures below.
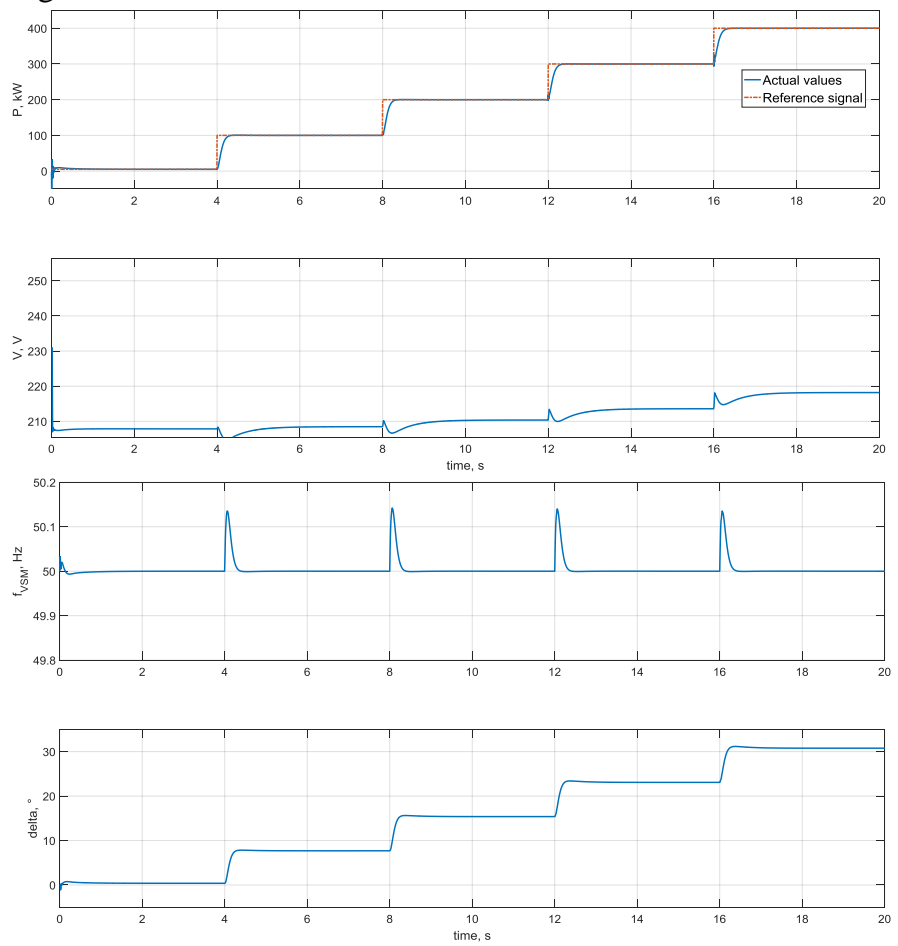

Fig. 6. Simulation results: active power, output voltage, VSM frequency and phase angle $\delta$

It can be observed that the LSD loop adjusts the voltage magnitude with acceptably small transients only.

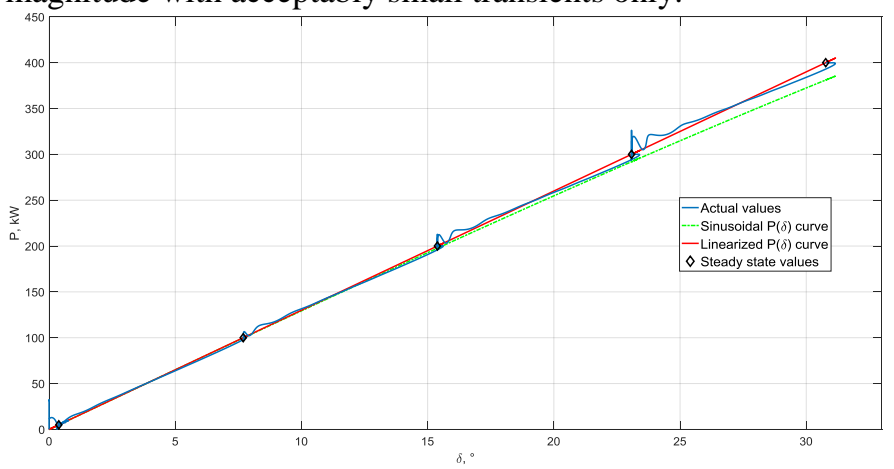

Fig. 7. Simulation results: $\mathrm{P}(\delta)$ curve transient and steady state values

It can also be observed that the steady-state $\mathrm{P}(\delta)$ value pairs are found at the linearized curve, according to the LSD control. 


\section{CONCLUSIONS}

This paper contributes to the discussion on the proper shaping of future power system dynamics, by defining appropriate requirements for the power electronic converters. We presented the new concept of Linearized and Uniform Swing Dynamics. This is a new control concept for power converters that implement VSMs. LSD control allows to linearize the inherently nonlinear power transmission equation, thereby allowing to shape the system eigenvalues so that they will be independent on the actual power level. This enables to use small-signal stability analysis technique for large signal stability analysis. Moreover, by properly choosing the controller parameters, the dynamics of the large number of converters expected in the system can be made unified and predictable. After deriving the control concept for two possible realizations (voltage reference control and virtual impedance control), simulation results are shown that demonstrate the controller operation.

Future work will include an in-depth stability analysis and the application of the concept in a multi-machine system.

\section{REFERENCES}

[1] Energy Concept 2050 for Germany with a European and Global Perspective, June 2010, http://www.fvee.de/fileadmin/publikationen/ Politische_Papiere_FVEE/10.06.Energy_Concept_2050/EK2010_EN.pdf

[2] https://www.h2020-migrate.eu/

[3] https://www.kopernikus-projekte.de/projekte/neue-netzstrukturen

[4] H. Bevrani,"Robust Power System Frequency Control", 2nd Edition, Springer International Publishing Switzerland, 2014.

[5] A. Benchaib,"Advanced Control of AC/DC Power Networks, system of systems approach based on spatio-temporal scales", John Wiley \& Sons Inc., Hoboken, USA, 2015.

[6] http://www.re-serve.eu/

[7] J. Machowski, J. W. Bialek, J. R. Bumby, "Power System Dynamics: Stability and Control," John Wiley \& Sons, Ltd., West Sussex, United Kingdom, 2008.

[8] J. Zhou and Y. Ohsawa, Improved swing equation and its properties in synchronous generators, Circuits and Systems I: Regular Papers, IEEE Transactions on Circuits and Systems, vol. 56, no. 1, pp. 200-209, Jan 2009.

[9] K. Visscher and S. W. H. De Haan, "Virtual synchronous machines (VSG's) for frequency stabilisation in future grids with a significant share of decentralized generation," CIRED Seminar 2008: SmartGrids for Distribution, Frankfurt, 2008, pp. 1-4.

[10] Q. C. Zhong, "Virtual Synchronous Machines: A unified interface for grid integration," in IEEE Power Electronics Magazine, vol. 3, no. 4, pp. 18-27, Dec. 2016. doi: 10.1109/MPEL.2016.2614906

[11] S. D'Arco and J. A. Suul, "Virtual synchronous machines Classification of implementations and analysis of equivalence to droop controllers for microgrids," 2013 IEEE Grenoble Conference, Grenoble, 2013, pp. 1-7. doi: 10.1109/PTC.2013.6652456

[12] O. Mo, S. D'Arco and J. A. Suul, "Evaluation of Virtual Synchronous Machines With Dynamic or Quasi-Stationary Machine Models," in IEEE Transactions on Industrial Electronics, vol. 64, no. 7, pp. 5952-5962, July 2017. doi: 10.1109/TIE.2016.2638810

[13] S. D'Arco, J. A. Suul and O. B. Fosso, "Control system tuning and stability analysis of Virtual Synchronous Machines," 2013 IEEE Energy Conversion Congress and Exposition, Denver, CO, 2013, pp. 2664-2671. doi: 10.1109/ECCE.2013.6647045

[14] S. D'Arco, J. A. Suul and O. B. Fosso, "Small-signal modelling and parametric sensitivity of a Virtual Synchronous Machine," 2014 Power Systems Computation Conference, Wroclaw, 2014, pp. 1-9. doi: 10.1109/PSCC.2014.7038410

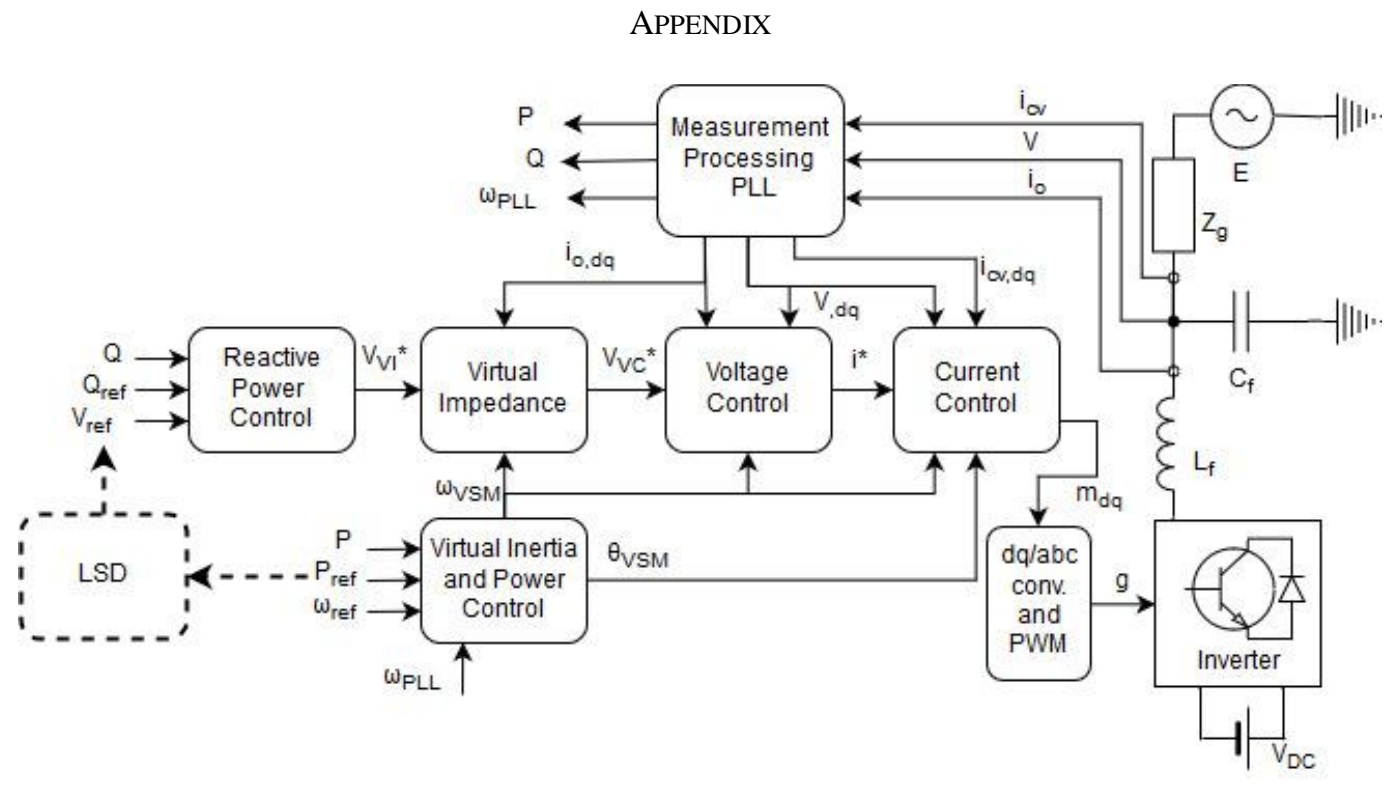

Fig. 8. Structure of the cascaded VSM control concept [14]

$$
\begin{gathered}
R_{v}=X \frac{\cos (2 \delta) * \operatorname{Im}(K)-\operatorname{Im}(K)+\delta k-\delta k \cos (2 \delta)-\delta k \operatorname{Re}(K)+\delta k \cos (2 \delta) \operatorname{Re}(K)+\delta k \sin (2 \delta) \operatorname{Im}(K)}{\cos (2 \delta)-2 \delta^{2} k^{2}+2 \delta k \sin (2 \delta)-1} \\
X_{v}=X \frac{\cos (2 \delta) * R e(K)-\operatorname{Re}(K)-2 \delta^{2} k^{2}+\delta k \sin (2 \delta)+\delta k \operatorname{Im}(K)+\delta k \sin (2 \delta) R e(K)-\delta k \cos (2 \delta) \operatorname{Im}(K)}{\cos (2 \delta)-2 \delta^{2} k^{2}+2 \delta k \sin (2 \delta)-1}
\end{gathered}
$$

where $k=(1-\varepsilon)$. 